\title{
Determinação eletroquímica de ciclamato de sódio em meio aquoso sobre filme de complexo de níquel
}

\author{
Vasconcellos, M. L. S. ${ }^{1 *}$; Buffon, E. ${ }^{1,2}$; Profeti, D.1; Profeti, L. P. R. ${ }^{1}$ \\ ${ }_{1}^{1}$ Departamento de Química e Física, Universidade Federal do Espírito Santo, Alegre, ES, Brasil. \\ ${ }^{2}$ Departamento de Química Analítica, Instituto de Química, Universidade Estadual Paulista, Araraquara, SP, Brasil. \\ * e-mail: Lourdes-vasconcellos@hotmail.com
}

\begin{abstract}
Resumo
Neste trabalho, um filme de hexacianoferrato de níquel (NiHCF) foi eletrodepositado sobre um eletrodo de carbono vítreo e empregado na eletroanálise de ciclamato de sódio. O NiHCF foi sintetizado por voltametria cíclica a partir de uma solução contendo $\mathrm{Ni}^{2+}, \mathrm{Fe}(\mathrm{CN})_{6}{ }^{3-}$ em solução aquosa de eletrólito. No perfil voltamétrico do NiHCF em solução de $\mathrm{KNO}_{3}$, foram observadas transições redox relacionadas às reações anódicas e catódicas do hexacianoferrato de níquel na presença do íon potássio. O eletrodo modificado mostrou uma boa resposta eletroquímica à molécula de ciclamato de sódio. A corrente de pico catódica em $0.56 \mathrm{~V}$ (vs. Ag/AgCl) no voltamograma cíclico diminuiu com o aumento da concentração do ciclamato. Os valores de corrente variaram linearmente com a concentração no intervalo de $5.0 \times 10^{-4}$ a $30.0 \times 10^{-4} \mathrm{~mol}$ $\mathrm{L}^{-1}$. Este resultado indica que o eletrodo modificado com NiHCF poderia ser empregado para a determinação de ciclamato de sódio.
\end{abstract}

\begin{abstract}
In this work, a film of nickel hexacyanoferrate (NiHCF) was electrodeposited on glassy carbon electrode and employed for the electro-analysis of sodium cyclamate. The NiHCF was synthesized by cyclic voltammetry from the mixing of $\mathrm{Ni}^{2+}, \mathrm{Fe}(\mathrm{CN})_{6}{ }^{3-}$ and electrolyte aqueous solutions. In the voltammetric profile of the NiHCF in $\mathrm{KNO}_{3}$ solution, redox transitions appeared related to anodic and cathodic reactions of nickel hexacyanoferrate(I/II) in presence of an electrolyte containing potassium ion. The modified electrode showed a good electrochemical response to sodium cyclamate molecule. The cathodic peak current at $0.56 \mathrm{~V}$ (vs. $\mathrm{Ag} / \mathrm{AgCl}$ ) in cyclic voltammogram decreased with the sodium cyclamate concentration. The current values varied linearly with the concentration in the range of $5.0 \times 10^{-4}$ to $30.0 \times 10^{-4} \mathrm{~mol} \mathrm{~L}^{-1}$. This result indicates that the NiHCF modified electrode could be used for the determination of sodium cyclamate.
\end{abstract}

Keywords (Palavras chaves): Eletrodo Modificado, Hexacianoferrato de Níquel, Ciclamato de Sódio

\section{Introdução}

Eletrodos Quimicamente Modificados (EQM's) são dispositivos formados por espécies quimicamente ativas imobilizadas em superfícies de eletrodos convencionais, como platina policristalina, carbono vítreo, ouro e mercúrio. Estes dispositivos podem ser empregados no desenvolvimento de métodos analíticos por possuírem vantagens frente aos eletrodos convencionais, tais como maior sensibilidade e seletividade [1]. 
A modificação química da superfície dos eletrodos pode ser feita através da incorporação simples de substâncias adsorventes. Dentre as espécies modificadoras existentes, há um destaque para os hexacianoferratos de metais (MHCF), os quais são análogos do Azul da Prússia (AP), e têm a capacidade de produzir filmes com propriedades redox específicas [2].

Estudos com EQM's têm contribuído amplamente para investigações em diversas áreas, como meio ambiente, saúde, biotecnologia, e indústria alimentícia. Como exemplo pode-se destacar o trabalho de Chen et al. [3], onde foi sintetizado um eletrodo modificado com hexacianoferrato de níquel e verificado sua aplicabilidade com base na voltametria cíclica e amperometria na determinação de ácido ascórbico em produtos farmacêuticos. Em outro trabalho, Suarez et al. [4] utilizou um eletrodo modificado com hexacianoferrato de cobre para a determinação de acetilcisteína em formulações farmacêuticas. Neste contexto nota-se que os EQM'S com MHCF têm apresentado diversas aplicabilidades [5].

Nos últimos anos as indústrias alimentícias vêm fazendo uso de diversos aditivos, dentre eles há uma relevância para dos edulcorantes artificiais (sacarina sódica, ciclamato de sódio, aspartame, acessulfame-K, entre outros) que são muito utilizados como adoçantes não calóricos em alimentos voltados para dietas especiais. $\mathrm{Na}$ classe dos edulcorantes podemos destacar o ciclamato de sódio, o qual é classificado como edulcorante não nutritivo artificial com alto poder adoçante, sendo aproximadamente $30-60$ vezes mais doce que a sacarose [5-6]. Devido a grande preocupação com relação à ingestão desse e de outros edulcorantes, principalmente por grupos específicos da população como crianças, diabéticos e obesos, os métodos analíticos são uma alternativa para o controle dos níveis de ciclamato de sódio e de outros produtos edulcorantes presentes em alimentos dietéticos [7]. Dentro deste contexto, o presente trabalho consistiu em sintetizar um filme de hexacianoferrato de níquel (NiHCF) sobre a superfície de um eletrodo de carbono vítreo (CV) e verificar a interação entre esse EQM e o aditivo alimentício ciclamato de sódio.

\section{Materiais e Métodos}

Para a realização deste trabalho o aparato experimental utilizado consistiu em uma célula eletroquímica contendo um eletrodo de trabalho de carbono vítreo (disco com $3 \mathrm{~mm}$ de diâmetro), um fio de platina como contra-eletrodo e um eletrodo de $\mathrm{Ag} / \mathrm{AgCl}$ como referência. Para todos os estudos eletroquímicos realizados utilizou-se um Potenciostato/Galvanostato AUTOLAB - PGSTAT $128 \mathrm{~N}$ controlado pelo software GPES 4.9.

Antes da formação do filme, o eletrodo de carbono vítreo foi polido com uma suspensão de alumina 0,05 $\mu \mathrm{m}$ e lavado com água ultrapura para a remoção das partículas. Após a limpeza mecânica, o eletrodo foi submetido a uma limpeza eletroquímica utilizando a técnica de voltametria cíclica entre os potencias $-1,0$ a $1,0 \mathrm{~V}$ VS. $\mathrm{Ag} / \mathrm{AgCl}$, com velocidade de varredura $50 \mathrm{mV}$ $\mathrm{s}^{-1}$ em uma solução de $0,1 \mathrm{~mol} \mathrm{~L}^{-1}$ de $\mathrm{KNO}_{3}$.

Para a preparação do EQM contendo NiHCF, a superfície do eletrodo de CV, previamente limpa, foi imersa numa célula eletroquímica contendo uma solução de $\mathrm{K}_{3} \mathrm{Fe}(\mathrm{CN})_{6} 1,0 \times 10^{-1} \mathrm{~mol} \mathrm{~L}^{-1}$ e uma solução de $\mathrm{Ni}\left(\mathrm{NO}_{3}\right)_{2} 1,0 \times 10^{-1} \mathrm{~mol} \mathrm{~L}^{-1}$. Ambas as soluções utilizadas foram preparadas numa solução eletrolítica de $\mathrm{KNO}_{3} 1,0 \times 10^{-1} \mathrm{~mol} \mathrm{~L}^{-1}$. O eletrodo de CV foi submetido a 40 ciclos sucessivos de varredura de potenciais, em um intervalo entre $-0,1$ a $0,8 \mathrm{~V}$ vs. $\mathrm{Ag} / \mathrm{AgCl}$, a uma velocidade de varredura de $100 \mathrm{mV} \mathrm{s}^{-1}$, para eletrodeposição do filme de NiHCF.

Os reagentes utilizados neste trabalho $\left(\mathrm{Ni}\left(\mathrm{NO}_{3}\right)_{2}\right.$, $\mathrm{K}_{3}\left[\mathrm{Fe}(\mathrm{CN})_{6}\right], \mathrm{KNO}_{3}$, e $\mathrm{C}_{6} \mathrm{H}_{12} \mathrm{NNaO}_{3} \mathrm{~S}$ foram de grau analítico e as soluções aquosas foram preparadas com água ultrapura obtido de um sistema de purificação MEGAPURITY MEGA RO/UP.

\section{Resultado e Discussão}

A primeira etapa deste trabalho consistiu na eletrossíntese do composto de coordenação hexacianoferrato de níquel (NiHCF) sobre a superfície do eletrodo de CV utilizando a técnica de voltametria cíclica, onde o eletrodo foi submetido a sucessivos ciclos entre os potenciais $-0,1 \mathrm{~V}$ a $0,8 \mathrm{~V}$ com velocidade de 
varredura a $100 \mathrm{mV} \mathrm{s}^{-1}$ na presença de $\mathrm{Ni}\left(\mathrm{NO}_{3}\right)_{2} \mathrm{e}$ $\mathrm{K}_{3}\left[\mathrm{Fe}(\mathrm{CN})_{6}\right]$, ambos na concentração de $1,0 \times 10^{-1} \mathrm{~mol}$ $\mathrm{L}^{-1}$ em solução eletrolítica $\mathrm{KNO}_{3} 1,0 \times 10^{-1} \mathrm{~mol} \mathrm{~L}^{-1}$. Os voltamogramas cíclicos, inicial e final, obtidos na etapa de síntese são mostrados na Figura 1.

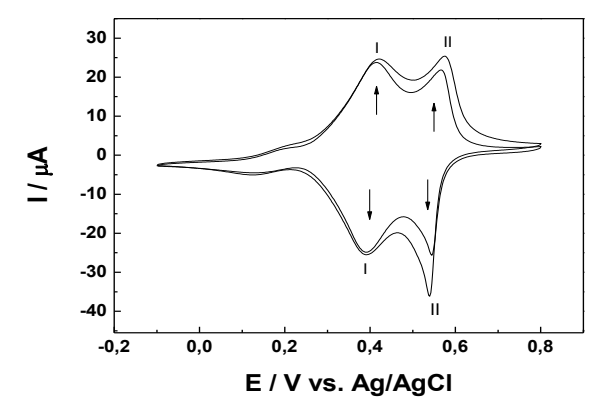

Figura1: Crescimento do filme NiHCF em KNO $31,0 \times 10^{-1} \mathrm{~mol} \mathrm{~L}^{-1}$ a 100 $\mathrm{mV} \mathrm{s}^{-1}$

Na Figura 1, observa-se que há o aumento das correntes dos picos catódicos e anódicos, indicando um processo de eletrodeposição sobre a superfície do eletrodo de CV. Estes picos indicam um comportamento de reversibilidade das reações redox que ocorrem na superfície do filme de NiHCF, as quais ocorrem em aproximadamente $0,40 \mathrm{~V}$ e 0,56 V. Essas reações redox são descritas pelas equações (1) e (2).

$$
\begin{aligned}
& \mathrm{K}_{2} \mathrm{Ni}^{\prime \prime}\left[\mathrm{Fe} \mathrm{CN}_{6}\right] \leftrightarrow \mathrm{KNi}^{\prime \prime}\left[\mathrm{Fe}^{\prime \prime \prime}(\mathrm{CN})_{6}\right]+\mathrm{e}^{-}+\mathrm{K}^{+} \\
& \mathrm{KNi}_{1.5}{ }^{\prime \prime}\left[\mathrm{Fe}^{\prime \prime}(\mathrm{CN})_{6}\right] \leftrightarrow \mathrm{Ni}_{1.5}^{\prime \prime}\left[\mathrm{Fe}^{\prime \prime \prime}(\mathrm{CN})_{6}\right]+\mathrm{e}^{-}+\mathrm{K}^{+}
\end{aligned}
$$

Nota-se, através das equações (1) e (2), a ocorrência da oxidação de $\mathrm{Fe}^{2+}$ a $\mathrm{Fe}^{3+}$ do grupo hexacianoferrato bem como o deslocamento do $\mathrm{K}^{+}$presente na estrutura do complexo [8]. Desta forma, a presença de dois pares de picos redox sugere a coexistência de duas formas estáveis de NiHCF, conforme descrito por Chen et al. [3].

A diferença existente entre os potenciais de pico referente a cada espécie de NiHCF, isto é, o par de picos redox localizado em $0,40 \mathrm{~V}$ (I) e o par de picos localizado em 0,56 V (II), pode ser atribuída a maior força de interação entre o íon potássio e o ferricianeto de níquel na espécie $\left.\mathrm{K}_{2} \mathrm{Ni}^{\prime \prime}\left[\mathrm{Fe}{ }^{\|} \mathrm{CN}\right)_{6}\right]$, sendo o maior potencial de pico referente à formação desta espécie [9]. O comportamento eletroquímico do complexo sintetizado possui características semelhantes àquelas descrita por
Chen et al.[3] e Castilho et al. [10], que descrevem que o complexo de hexacianoferrato de níquel possui estrutura cristalina cúbica, onde os átomos metálicos se encontram ligados por grupos cianetos. Nesta estrutura, todos os átomos de ferro se coordenam aos átomos de carbono do grupo cianeto, enquanto que todos os átomos de níquel se coordenam aos átomos de nitrogênio, onde os centros de ferro são reversíveis e que alternam entre $\mathrm{Fe}^{3+}$ e $\mathrm{Fe}^{2+}$ garantindo assim a eletroneutralidade do composto [3 - 10].

O estudo de estabilidade do filme de NiHCF foi realizado com o EQM imerso em solução de $\mathrm{KNO}_{3} 1,0 \times 10^{-1} \mathrm{~mol}$ $\mathrm{L}^{-1}$ aplicando-se 400 ciclos sucessivos de varredura de potenciais numa velocidade de varredura de $100 \mathrm{mV} \mathrm{s}$ 1. Os resultados obtidos com esse estudo são apresentados na Figura 2.

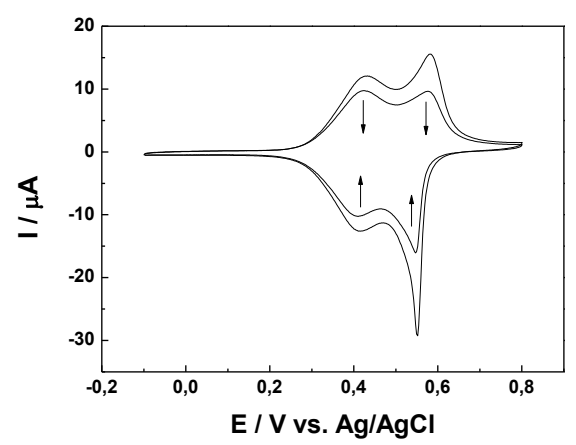

Figura 2: Estabilidade do filme de NiHCF em solução $\mathrm{KNO}_{3} 1,0 \times 10^{-1}$ mol L-1 com velocidade de varredura de $100 \mathrm{mV} \mathrm{s}^{-1}$.

O voltamograma do filme de NiHCF imobilizado sobre o eletrodo de CV obtido após 400 ciclos apresentou um decréscimo de 19,66\% na intensidade de corrente de pico, fato que pode ser atribuído à dissolução gradual do complexo na solução eletrolítica de $\mathrm{KNO}_{3}$.

A fim de verificar interação do filme de NiHCF com a molécula de ciclamato de sódio, investigou-se 0 comportamento eletroquímico desta molécula com o EQM. A resposta eletroquímica obtida está representada na Figura 3. 


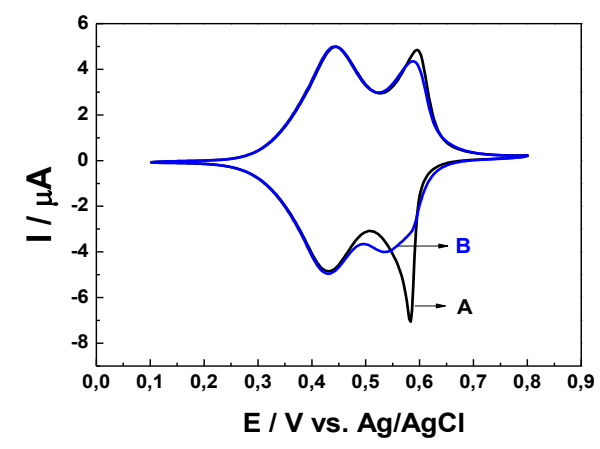

Figura 3: Perfil voltamétrico do filme de NiHCF na (A) presença de solução eletrolítica $\mathrm{KNO}_{3} 1,0 \times 10^{-1} \mathrm{~mol} \mathrm{~L}^{-1}$ e na (B) presença de solução eletrolítica de $\mathrm{KNO}_{3} 1,0 \times 10^{-1} \mathrm{~mol} \mathrm{~L}^{-1}+$ solução de ciclamato de sódio 10 $\times 10^{-3} \mathrm{~mol} \mathrm{~L}^{-1}$, numa velocidade de varredura de $20 \mathrm{mV} \mathrm{s}^{-1}$.

Na Figura 3 verifica-se que o perfil ciclovoltamétrico do filme de NiHCF foi modificado pela presença do ciclamato de sódio, sendo observado a diminuição da corrente de pico catódica no potencial de aproximadamente $0,56 \mathrm{~V}$. Tal alteração no perfil voltamétrico indica que há interação entre o filme de $\mathrm{NiHCF}$ com o aditivo alimentar.

Desta forma, para fins comparativos, verificou-se o perfil voltamétrico do filme de NiHCF na presença de ciclamato de sódio em diferentes concentrações. Os resultados obtidos com esse estudo são apresentados pelas Figuras 4 e 5.

Na Figura 4, observa-se o comportamento eletroquímico do filme de NiHCF na presença de ciclamato de sódio em diferentes concentrações, onde nota-se que a corrente catódica no potencial de $0,56 \mathrm{~V}$ apresentou diminuição da intensidade conforme o aumento da concentração do ciclamato de sódio. Esta variação da intensidade da corrente pode ser atribuída à interação entre o complexo NiHCF e o átomo de enxofre presente na estrutura da molécula de ciclamato de sódio (Figura 6) [11].

Ao analisar a Figura 5, observa-se que os valores da corrente de pico catódica (Ipc) variam linearmente com a concentração de ciclamato de sódio de acordo com a seguinte equação:

Ipc $(A)=-7,33531 \times 10^{-6}+4,98269 \times 10^{-4} \mathrm{c}\left(\mathrm{mol} \mathrm{L}^{-1}\right) ; \mathrm{com}$ um coeficiente de correlação linear (R2) de 0,99991.

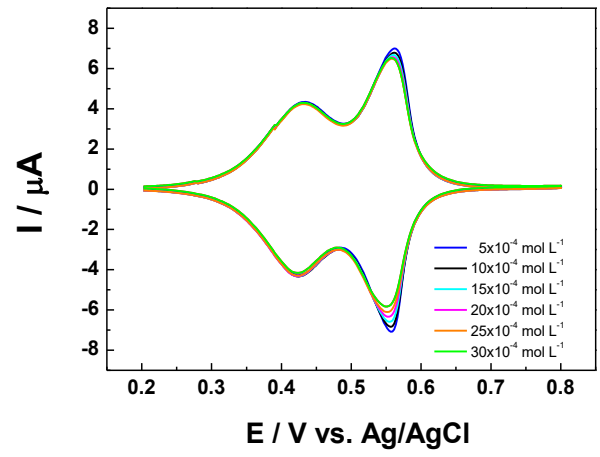

Figura 4: Comportamento da corrente de pico catódica no potencial de $0,56 \mathrm{~V}$, na presença de diversas soluções de ciclamato de sódio em diferentes concentrações.

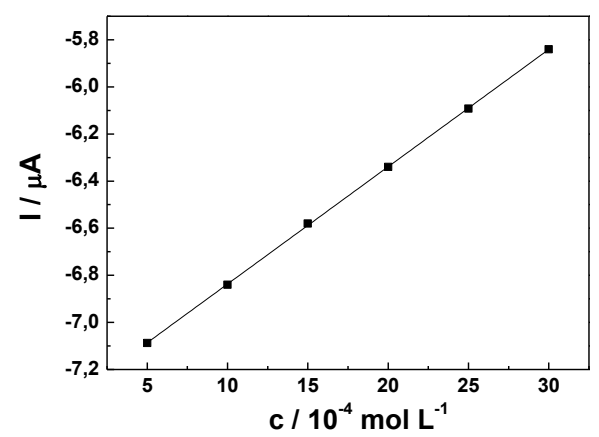

Figura 5: Relação entre as concentrações de ciclamato de sódio e os valores das correntes de pico catódica do EQM contendo NiHCF.

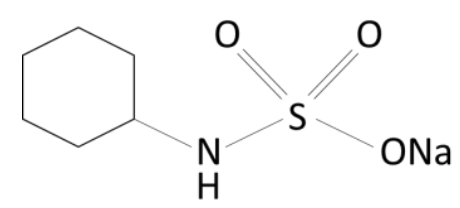

Figura 6: Estrutura molecular do ciclamato de sódio

A relação linear observada no comportamento eletroquímico no filme de NiHCF entre as correntes de pico catódica vs. as concentrações de ciclamato de sódio indicam que uma resposta analítica pode ser obtida da interação entre esse EQM e a molécula de ciclamato de sódio, a qual indica a possibilidade de desenvolvimento de um método analítico promissor para a detecção desse aditivo alimentar em produtos dietéticos. 


\section{Conclusões}

A técnica de voltametria cíclica utilizada neste trabalho possibilitou a síntese do filme de NiHCF sobre a superfície do eletrodo de CV e a verificação da interação desse EQM com a molécula de ciclamato de sódio. O comportamento eletroquímico apresentado pela molécula de ciclamato de sódio indicou a potencial aplicação desse EQM no desenvolvimento de um método analítico especifico para a determinação desse composto em produtos alimentícios.

\section{Agradecimentos}

Os autores agradecem à Fundação de Amparo à Pesquisa do Espírito Santo (FAPES) e ao Programa Institucional de Iniciação Cientifica (PIIC) da Universidade Federal do Espirito Santo.

\section{Referências}

[1] - QUINTINO, M. S. M. Desenvolvimento de sensores eletroquímicos associados a batch injection analysis (bia) para aplicações analíticas, 2003, 112f, Tese (Doutorado em Ciências - Química Analítica) - Instituto de Química, Universidade de São Paulo, 2003.

[2] GANESH, V.; MAHESWARI, D. L.; BERCHMANS, S. Electrochimica Acta, v.56, p.1197-1207, 2011.

[3] CHEN, W.; TANG, J.; CHENG, H-J.; XIA, X-H. Talanta, v. 80, p. 539-543, 2009.
[4] SUAREZ, W.T; MARCOLINO Jr., L.H.; FATIBELOFILHO, O., Microchemical Journal, v. 82, p.163-167, 2006.

[5] CHANG, C.-S.; YEH, T. S., Journal of Food and Drug Analysis, v. 22, p. 318-322, 2014.

[6] CAMARGo, M. C. R.; TOledo, M. C. F. Revista Instituto Adolfo Lutz, v.65, p.100-105, 2006.

[7] ZYGLER, A.; WASIK, A.; NAMIES'NIK J. Trends in Analytical Chemistry, v. 28, No. 9, 2009.

[8] KARNJANAKOM, S.; GUAN, G.; PHANTHONG, P.; HAO, X.; DU, X.; SAMART, C.; ABUDULA, A. Electrochimica Acta, v.139, p.36-41, 2014.

[9] ABBASPOUR, A.; KHAJEHZADEH, A., GHAFFARINEJAD. A. Journal of Electroanalytical Chemistry, v. 631, p. 52-57, 2009.

[10] CRESPILHO, F. N. Membranas eletroativas nanoestruturadas: Estudo de transporte de carga e imobilização Enzimática, 2007, 130f, Tese (Doutorado em ciências- físico-química) - Universidade Federal de São Carlos, 2007.

[11] MILANI, M.R.; STRADIOTTO, N.R.; CARDOSO, A. A., Electroanalysis, v. 15, p. 827-830, 2003. 